\title{
Tipps und Tricks: \\ Anatomiegerechte Reposition von Densfrakturen
}

$\square$ Daniel Krüßmann, Thomas Ruffing
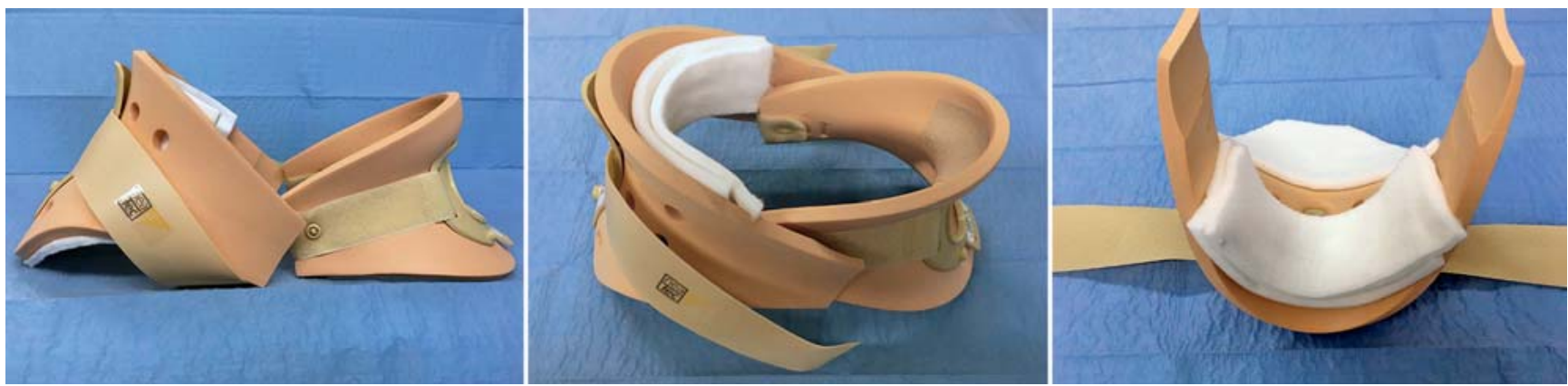

Abb. 1 Maßgeschneiderte Anwendung an der festen Zervikalstütze. Reposition durch Distraktion und Flexion aufgrund der Polsterapplikation kaudal und dorsal.
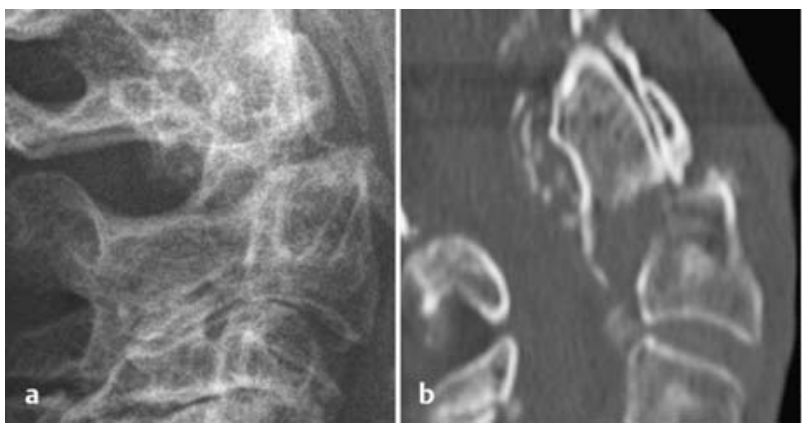

Abb. 2 a bis c Frakturstellung in Zervikalstütze ohne (a, b) und mit (c) Polsterung.

Densfrakturen werden bis zur definitiven operativen Versorgung meist in einer Zervikalstütze ruhiggestellt. Je nach Anatomie des Unfallverletzten bzw. Frakturdislokation besteht aufgrund der dadurch wenig rigiden Anordnung die
Gefahr der Dislokation und damit der Myelonschädigung. Unter Zuhilfenahme von einseitig klebendem Polstermaterial (z.B. Cellona, Fa. Lohmann \& Rauscher, Neuwied), welches normalerweise bei Cast- oder Gipsversorgungen von Extre- mitätenfrakturen Anwendung findet, kann die Orthese maßgeschneidert an die jeweilige Anatomie bzw. Frakturdislokation angepasst werden. Somit wird eine sanfte Reposition der Fraktur erzielt. Die Bilder zeigen beispielhaft die Versorgung einer Extensionsfraktur.

\section{Daniel Krüßmann \\ Assistenzarzt \\ Dr. med. Thomas Ruffing \\ Oberarzt}

Klinik für Unfallchirurgie und

Orthopädie 1

Westpfalz-Klinikum GmbH

Hellmut-Hartert-Straße 1

67655 Kaiserlautern

danielkruessmann@gmx.de 